Barbara Baragatti, Michal Laniado Schwartzman, Debora Angeloni, Francesca Scebba, Enrica Ciofini, Daria Sodini, Virginia Ottaviano, Simona Nencioni, Aldo Paolicchi, Joan P. Graves, Darryl C. Zeldin, Katherine Gotlinger, Stefano Luin and Flavio Coceani

Am J Physiol Heart Circ Physiol 297:2161-2168, 2009. First published Oct 2, 2009;

doi:10.1152/ajpheart.00576.2009

You might find this additional information useful...

Supplemental material for this article can be found at:

http://ajpheart.physiology.org/cgi/content/full/00576.2009/DC1

This article cites 35 articles, 22 of which you can access free at:

http://ajpheart.physiology.org/cgi/content/full/297/6/H2161\#BIBL

Updated information and services including high-resolution figures, can be found at:

http://ajpheart.physiology.org/cgi/content/full/297/6/H2161

Additional material and information about AJP - Heart and Circulatory Physiology can be found at: http://www.the-aps.org/publications/ajpheart

This information is current as of September 21, 2010 .

AJP - Heart and Circulatory Physiology publishes original investigations on the physiology of the heart, blood vessels, and 


\title{
EDHF function in the ductus arteriosus: evidence against involvement of epoxyeicosatrienoic acids and 12S-hydroxyeicosatetraenoic acid
}

\author{
Barbara Baragatti, ${ }^{1,2}$ Michal Laniado Schwartzman, ${ }^{3}$ Debora Angeloni, ${ }^{1,2}$ Francesca Scebba, ${ }^{1}$ \\ Enrica Ciofini, ${ }^{1}$ Daria Sodini, ${ }^{1}$ Virginia Ottaviano, ${ }^{4}$ Simona Nencioni, ${ }^{1}$ Aldo Paolicchi, ${ }^{4}$ Joan P. Graves, ${ }^{5}$ \\ Darryl C. Zeldin, ${ }^{5}$ Katherine Gotlinger, ${ }^{3}$ Stefano Luin, ${ }^{6}$ and Flavio Coceani ${ }^{1,2}$ \\ ${ }^{1}$ Scuola Superiore Sant'Anna, and ${ }^{2}$ Institute of Clinical Physiology Consiglio Nazionale delle Ricerche, Pisa, Italy; \\ ${ }^{3}$ Department of Pharmacology, New York Medical College, Valhalla, New York; ${ }^{4}$ Department of Experimental Pathology, \\ University of Pisa, Pisa, Italy; ${ }^{5}$ Division of Intramural Research, National Institute of Environmental Health Sciences, \\ National Institutes of Health, Research Triangle Park, North Carolina; and ${ }^{6}$ National Enterprise for nanoScience \\ and nanoTechnology, Scuola Normale Superiore/Consiglio Nazionale delle Ricerche-Istituto Nazionale per la Fisica della \\ Materia, Pisa, Italy
}

Submitted 29 June 2009; accepted in final form 29 September 2009

\begin{abstract}
Baragatti B, Schwartzman ML, Angeloni D, Scebba F, Ciofini E, Sodini D, Ottaviano V, Nencioni S, Paolicchi A, Graves JP, Zeldin DC, Gotlinger K, Luin S, Coceani F. EDHF function in the ductus arteriosus: evidence against involvement of epoxyeicosatrienoic acids and 12S-hydroxyeicosatetraenoic acid. Am J Physiol Heart Circ Physiol 297: H2161-H2168, 2009. First published October 2, 2009; doi:10.1152/ajpheart.00576.2009.-We have previously shown (Ref. 2) that endothelium-derived hyperpolarizing factor (EDHF) becomes functional in the fetal ductus arteriosus on removal of nitric oxide and carbon monoxide. From this, it was proposed that EDHF originates from a cytochrome $P-450$ (CYP450)-catalyzed reaction being inhibited by the two agents. Here, we have examined in the mouse ductus whether EDHF can be identified as an arachidonic acid product of a CYP450 epoxygenase and allied pathways. We did not detect transcripts of the mouse CYP2C subfamily in vessel, while CYP2J subfamily transcripts were expressed with CYP2J6 and CYP2J9. These CYP2J hemoproteins were also detected in the ductus by immunofluorescence microscopy, being colocalized with the endoplasmic reticulum in both endothelial and muscle cells. Distinct CYP450 transcripts were also detected and were responsible for $\omega$-hydroxylation (CYP4A31) and 12R-hydroxylation (CYP4B1). Mass spectrometric analysis showed formation of epoxyeicosatrienoic acids (EETs) in the intact ductus, with 11,12- and 14,15-EETs being more prominent than 5,6- and 8,9-EETs. However, their yield did not increase with nitric oxide/carbon monoxide suppression, nor did it abate with endothelium removal. No evidence was obtained for formation of $12 \mathrm{R}$-hydroxyeicosatrienoic acid and $\omega$-hydroxylation products. 2S-hydroxyeicosatetraenoic acid was instead detected, and, contrary to data implicating this compound as an alternative EDHF, its suppression with baicalein did not modify the EDHF-mediated relaxation to bradykinin. We conclude that none of the more common CYP450-linked arachidonic acid metabolites appears to qualify as EDHF in mouse ductus. We speculate that some novel eicosanoid or a totally unrelated compound requiring CYP450 for its synthesis accounts for EDHF in this vessel.
\end{abstract}

bradykinin; arachidonic acid epoxygenase; arachidonic acid monooxygenase; fetal and neonatal physiology

SEVERAL DIFFERENT COMPOUNDS may sustain the active relaxation of blood vessels, and their relative importance varies with the district and prevailing physiological or pathophysiological

Address for reprint requests and other correspondence: F. Coceani, Scuola Superiore Sant'Anna, Piazza Martiri della Libertà 33, 56127 Pisa, Italy (e-mail: coceani@sssup.it). condition. Among them, endothelium-derived hyperpolarizing factor (EDHF) has attracted much attention due to its elusive nature (10). Indeed, a host of agents have been identified that possess this particular activity, but none of them meets with general acceptance (10). Nevertheless, a strong case can be made for the cytochrome $P$-450 (CYP450)-derived metabolites of arachidonic acid (AA), specifically those formed via the epoxygenase pathway [i.e., epoxyeicosatrienoic acids (EETs)] $(4,29)$. For example, Huang et al. (13) have reported a causal relationship between EET release and the EDHF response. Furthermore, the same response has been linked with CYP2C hemoproteins (11), which, together with CYP2J, represent the primary catalytic enzymes for AA epoxidation within the vasculature $(12,29)$. Germane to this subject is also the postulated vasodilator function at certain sites of products of bis-allylic (lipoxygenase-like) oxidation and $\omega$-hydroxylation, specifically 12R-hydroxyeicosatrienoic (12R-HETrE) and 20-hydroxytetraenoic acids (20-HETE), whose catalytic hemoproteins belong to CYP4A and CYP4B subfamilies (19-21).

In a previous investigation, our laboratory has found that EDHF function is normally absent in the ductus arteriosus, but becomes evident following the combined suppression of nitric oxide (NO) and carbon monoxide $(\mathrm{CO})$ relaxing mechanisms (2). Based on this observation, the concept was put forward that $\mathrm{NO}$ and $\mathrm{CO}$ exert tonic inhibition of a CYP450-catalyzed reaction, yielding one or more EDHFs. By extension, the activity of this reaction was considered critical in determining the state, latent vs. overt, of the EDHF mechanism.

From this premise, the objective of the present study was twofold: 1) to assess the presence in the mouse ductus of CYP450 species potentially relevant to EDHF biosynthesis; and 2) to determine whether AA metabolite(s), specifically EET(s), qualify as EDHF in this vessel. In the process, however, the analysis was extended to candidates for EDHF function not originating from a CYP450 pathway (i.e., from AA 12S-lipoxygenase; see Refs. 10, 36) as well as to non-EDHFtype AA products from a CYP450-catalyzed reaction with the potential to sustain relaxation $(20,21)$. As in the past, we used the ductus from the mouse, which, by lacking vasa vasorum (26) and any active compound formed therein, is eminently suited for the study of EDHF and allied, endothelium-based relaxants. 


\section{MATERIALS AND METHODS}

Experiments were carried out in wild-type C57BL/6 mice (Harlan, San Pietro al Natisone, Italy) (litter size, 4-13). Animals were housed in temperature- and humidity-controlled quarters, with constant 12: 12-h light-dark cycles, and were given food and water ad libitum. Surgical procedures and experimental protocols were approved by the Animal Care Committee of the Ministry of Health.

Solutions and drugs. The Krebs medium had the following composition $(\mathrm{mM})$ : $118 \mathrm{NaCl}, 4.7 \mathrm{KCl}, 2.5 \mathrm{CaCl}_{2}, 1 \mathrm{KH}_{2} \mathrm{PO}_{4}, 0.9$ $\mathrm{MgSO}_{4}, 11.1$ dextrose, and $25 \mathrm{NaHCO}_{3}$. Depending on protocol and stage of the experiment, the solution was bubbled with gas mixtures containing either no $\mathrm{O}_{2}$ or $\mathrm{O}_{2}$ in one of two concentrations (2.5 and $30 \%$ ) plus $5 \% \mathrm{CO}_{2}$ and balance $\mathrm{N}_{2}$. The $2.5 \%$ concentration was chosen to mimic the fetal condition, while the $30 \%$ concentration aimed to promote oxygen-dependent processes and CYP450-catalyzed reactions in particular. $\mathrm{PO}_{2}$ of the medium was measured with a Chiron gas analyzer (model 248, Halstead, UK) and was $1.16 \pm 0.01$, $2.21 \pm 0.02$, and $20.9 \pm 0.1 \mathrm{kPa}(\mathrm{pH} 7.42 \pm 0.004)$ when gas mixtures had $0,2.5$, and $30 \% \mathrm{O}_{2}$, respectively.

Polyclonal CYP2J6 and CYP2J9 antisera were generated in the rabbit, as described $(18,24)$. Their capability to uniquely recognize the corresponding mouse isoforms was confirmed in control experiments, which also excluded any coupling with isoforms of the CYP2C, CYP4A, or CYP4B subfamilies $(18,24)$.

The following compounds were used: bradykinin acetate (Sigma, St. Louis, MO); the NO synthase inhibitor, $N^{\mathrm{G}}$-nitro-L-arginine methyl ester (L-NAME, Sigma); the heme oxygenase inhibitor, zinc protoporphyrin (ZnPP, Porphyrin Products, Carnforth, UK); the dual cyclooxygenase (COX) 1 and 2 inhibitor, indomethacin (Sigma); the recently developed soluble epoxide hydrolase inhibitor 1-trifluoromethoxyphenyl-3-(1-acetylpiperidin-4-yl)urea (also known as compound 1555 , courtesy of Dr. B. D. Hammock) (14); the 12S-lipoxygenase inhibitor, baicalein (Sigma); the thromboxane $\mathrm{A}_{2}\left(\mathrm{TxA}_{2}\right)$ analog 9,11-epithio-11,12-methano- $\mathrm{TxA}_{2}$ (ONO-11113, courtesy of ONO Pharmaceutical, Osaka, Japan); and retinoic acid (all-trans-retinol palmitate, Sigma). Concentrations of the inhibitors were derived from the literature with the aim of combining efficacy with selectivity.

ONO-11113 was dissolved in distilled ethanol $(5 \mathrm{mg} / \mathrm{ml})$, and aliquots (stored at $-70^{\circ} \mathrm{C}$ ) were diluted with Tris buffer ( $\mathrm{pH} 7.4$ ). Indomethacin and baicalein were also dissolved in ethanol (10 and $1.35 \mathrm{mg} / \mathrm{ml}$, respectively) before the final solution was prepared in the Krebs medium. Likewise, ZnPP was first prepared as a stock solution in $0.1 \mathrm{M} \mathrm{NaOH}(1 \mathrm{mM})$ on the day of the experiment. Retinoic acid was instead diluted in safflower seed oil. Ethanol in fluid bathing ductus preparations did not exceed $0.2 \%$ (baicalein), $0.01 \%$ (indomethacin), or $0.001 \%$ (ONO-11113). Other substances dissolved readily in Krebs medium. Solutions of $\mathrm{ZnPP}$ and retinoic acid were protected from light.

Concentrations of compounds are given in molar units and refer to their final value in Krebs medium.

General procedure. Term fetal mice (gestational age, 19 days) were delivered by cesarean section under halothane anesthesia and were killed by cervical dislocation. Selected experiments were also performed in the preterm animal (gestational age, 17 days), with and without prior treatment with retinoic acid to the mother. Retinoic acid, a known promoter of structural and functional maturation in the ductus arteriosus $(32,35)$, was given by intramuscular route $(1 \mathrm{mg} / \mathrm{kg}$ equivalent to $1,818 \mathrm{IU}$ ) for 3 consecutive days before the experiment. This dose had been chosen after confirming, by semiquantitative RT-PCR, its effectiveness in upregulating CYP26A1 expression in the liver of the mother (25) (Supplemental Fig. 1; the online version of this article contains supplemental data). The procedure for dissection of the ductus has been described previously (8). In brief, the animal was secured with its left side up in a dissection chamber containing ice-cold Krebs solution gassed with $5 \% \mathrm{CO}_{2}$ in $\mathrm{N}_{2}$. Through a thoracotomy, the ductus was exposed, separated from the adjoining large blood vessels, and then handled differently, depending on the protocol. In some experiments, the ductus was prepared without the endothelium. For this purpose, a cat whisker of suitable size, its surface coarse from polishing with fine-grain sandpaper (600 grit), was passed through the lumen before the vessel was isolated. The procedure for preparing endothelium-denuded, small-caliber vessels has been described elsewhere, and successful removal was confirmed by transmission electron microscopy and functional tests $(2,31)$.

Incubation experiments. Freshly dissected specimens of ductus arteriosus, intact or endothelium denuded, were pooled in a tightly sealed tube ( 8 per tube) containing $0.5 \mathrm{ml}$ of Krebs solution. The medium had been preequilibrated with a gas mixture containing $2.5 \%$ $\mathrm{O}_{2}$, and the same mixture was passed through the tube before starting the incubation. A gas mixture with $30 \% \mathrm{O}_{2}$ was used in few experiments. Depending on the protocol, the medium had no additions or contained bradykinin $(0.1 \mu \mathrm{M})$ plus an inhibitory cocktail (indomethacin $2.8 \mu \mathrm{M}$; L-NAME $100 \mu \mathrm{M}$; ZnPP $10 \mu \mathrm{M}$ ), ensuring the preferential formation of EDHF (2). Compound $1555(0.5 \mu \mathrm{M})$ was added alone or in combination with the cocktail. Baicalein $(10 \mu \mathrm{M})$ was also used in separate experiments. In all cases, incubation was carried out with gentle shaking for $1 \mathrm{~h}$ at $37^{\circ} \mathrm{C}$ and was terminated by adding methanol $(1 \mathrm{ml})$. Afterwards, the whole sample, comprising fluid and specimens, was frozen in an acetone-solid $\mathrm{CO}_{2}$ bath and was subsequently stored at $-80^{\circ} \mathrm{C}$ until analysis.

In vitro recording. Once isolated, the ductus arteriosus was suspended onto $25-\mu \mathrm{m}$ tungsten wires (Cooner wire, Chatsworth, CA) and placed inside an organ bath. The fluid was gassed with a gas mixture containing $2.5 \% \mathrm{O}_{2}$, and the same mixture was flushed through a hood covering the bath. Preparations were then equilibrated $\left(\sim 60 \mathrm{~min}\right.$ at $\left.37^{\circ} \mathrm{C}\right)$ with a minimum stretch being applied $(0.09 \pm$ $0.01 \mathrm{mN} / \mathrm{mm}, n=8)$ and the attendant internal circumference $\left(\mathrm{C}_{0}\right)$, with the related resting dimension served as a reference for choosing the appropriate load. Values for the resting dimension $(n=8)$ were as follows: internal diameter, $131 \pm 2 \mu \mathrm{m}$; length, $558 \pm 21$ and $568 \pm$ $19 \mu \mathrm{m}$ for the short and long side, respectively; and wall thickness, $21 \pm 0.5 \mu \mathrm{m}$. Afterwards, tension was applied to attain an operating circumference (i.e., $\mathrm{C}_{50}$ ), coinciding with the condition in vivo $(0.44 \pm 0.01 \mathrm{mN} / \mathrm{mm}, n=8)$, and the actual experiment was started following a second, 70- to 115 -min period of equilibration.

Intuitively, a prime aim should have been to test an epoxygenase inhibitor [i.e., N-methylsulfonyl-6-(2-propargyloxyphenol)hexanamide (MS-PPOH); see Ref. 30] on the bradykinin-induced relaxation under conditions favoring the selective formation of EDHF. However, this proved not to be feasible, because the inhibitor $(5$ or $10 \mu \mathrm{M})$ interfered with the ductus precontraction (B. Baragatti and F. Coceani, unpublished observations). ${ }^{1}$ Hence, the study was limited to the assessment of baicalein action on the EDHF response. For this purpose, preparations were precontracted with the inhibitory cocktail (see above), without and with the addition of baicalein $(10 \mu \mathrm{m})$, and bradykinin was applied to the bath in cumulative concentrations $(0.01$ $\mathrm{nM}-10 \mu \mathrm{m})$.

Total RNA preparation, RT-PCR, and real-time PCR. Ductuses were collected from term and preterm (without/with retinoic acid pretreatment) fetuses and were pooled in one group for each condition (30-40 specimens/group). Specimens of adult liver, kidney, or brain were used as a reference, depending on the specific gene under examination. Experiments in preterm animals were performed to ascertain whether, in accord with other ductus relaxing mechanisms,

\footnotetext{
${ }^{1}$ The finding is not altogether surprising, since a large body of evidence implicates a, hitherto unknown, CYP450-based reaction in the generation of contractile tone in the ductus (7). Quite appropriately, among the supporting elements is the demonstration of the relaxant action of CYP450 inhibitors of diverse chemical structure (7). Hence, there may be two CYP450 pathways operational in the vessel, involving distinct hemoproteins and sustaining opposite functions.
} 
Table 1. Gene expression profile for $C Y P 2 C, C Y P 2 J$, CYP4A, and CYP4B subfamilies in the mouse ductus arteriosus

$$
\text { CYP450 }
$$
mRNA Expression

CYP2C29, -37, -38, -39, -40, -44, -54, -55, -66, -70

CYP2J5, -8, -11, -12, -13

CYP2J6, -9

CYP4A10, -12, $-14,-29,-32$

CYP4A31*

CYP4B1

-
-
+
-
+
+

CYP450, cytochrome $P-450$. *CYP4A31 has $89 \%$ homology with rat CYP4A1, which is a prime element for arachidonic acid $\omega$-hydroxylation.

any CYP450 involved in EDHF function was expressed early in gestation. RNA was isolated, as previously described (1), with Tripure isolation reagent (Roche, Indianapolis, IN), and its yield was measured spectrophotometrically.

DNase I (Roche)-treated total RNA was reverse transcribed with 1 unit of thermoscript RT (Invitrogen, Carlsbad, CA) in the presence of random hexanucleotide primers. The cDNA product $(0.1$ or $0.2 \mu \mathrm{g}$ for each group) was used for amplification in semiquantitative and then comparative real-time PCR for selected genes. For the former analysis, primer sequences were either original or found in the literature (see Supplemental Table 1), while, for the latter, they were obtained from an online library (Applied Biosystems, Foster City, CA). For semiquantitative analysis, electrophoresis of PCR products was performed on agarose gels $(1-1.5 \% \mathrm{~W} / \mathrm{vol}$, depending on the length of the amplified fragment) stained with ethidium bromide, and bands from the ductus and reference organs were visualized in parallel. Quantitative real-time PCR reactions (40 cycles) were performed on an ABI Prism 7700 instrument using the TaqMan Universal PCR mastermix (Applied Biosystems). Gene expression was quantified in triplicate by the comparative cycle threshold method (ABI Prism 7700 Relative Quantification Software, Applied Biosystems) and was expressed in arbitrary units against reference $\beta$-actin.

Immunohistochemistry. Specimens of ductus arteriosus that had been frozen in an embedding medium (Tissue-Tek optimum cutting temperature compound; Sakura Finetek, Torrance, CA) were cut serially (5- $\mu \mathrm{m}$-thick sections) in a transversal plane. Sections were fixed in cold acetone $\left(10 \mathrm{~min}\right.$ at $\left.-20^{\circ} \mathrm{C}\right)$ and, for antigen retrieval, were then immersed in citrate buffer $(0.01 \mathrm{M}, \mathrm{pH}$ 6.0) for a 15-min treatment in a microwave oven. Afterwards, they were kept in goat serum $(10 \% \mathrm{vol} / \mathrm{vol})$ for $1 \mathrm{~h}$ at room temperature, and this was followed by incubation overnight with the CYP2J6 or CYP2J9 antibody (dilution, 1:250 for both) at $4{ }^{\circ} \mathrm{C}$. Goat anti-rabbit IgG conjugated to FITC (Zymed, Carlsbad, CA) (dilution, 1:100) served as secondary antibody. Specificity of binding was verified by omitting the primary antibody.

Colocalization of immunoreactive sites with the plasma membrane and the endoplasmic reticulum was ascertained through incubation with appropriate markers (wheat germ agglutinin conjugates marine blue and BODIPY 558/568 Brefeldin A, respectively; Molecular Probes, Eugene, OR), according to the manufacturer's instructions.

In all cases, slides for analysis were protected with an anti-fading agent (Vectashield; Vector, Burlingame, CA). Images were acquired on a fluorescence (Leica mod DMR) or confocal (Leica TCS-SP2 on mod DM IRE 2) microscope, as required.

Solid-phase extraction of eicosanoids. Analysis was performed on individual samples and, exceptionally, on five samples combined (i.e., 40 specimens). In either case, frozen incubates were thawed on ice and, after adding a host of compounds $\left(\left[{ }^{2} \mathrm{H}_{4}\right] \mathrm{PGE}_{2} ; 8,9-\left[{ }^{2} \mathrm{H}_{11}\right]\right.$ EET or 11,12- $\left[{ }^{2} \mathrm{H}_{8}\right]$ EET; 12- $\left[{ }^{2} \mathrm{H}_{8}\right]$ HETE; 20- $\left[{ }^{2} \mathrm{H}_{6}\right]$ HETE; 11,12- $\left[{ }^{2} \mathrm{H}_{11}\right] \mathrm{di}-$ HETrE) as internal standards for recovery, were centrifuged for 15 $\min$ at $4{ }^{\circ} \mathrm{C}$. The resultant pellet was used for protein measurement by a Bradford method (BioRad reagent, catalog no. 500-0006; Hercules,
CA), while the supernatant was collected, diluted with water $(15 \mathrm{ml})$, and acidified to $\mathrm{pH} 4.0$ with $\mathrm{HCl}$. Samples were then applied to a $\mathrm{C}_{18}$-ODS AccuBond II $500 \mathrm{mg}$ cartridge (Agilent Technologies, Santa Clara, CA) that had been preconditioned with methanol and water (20 $\mathrm{ml}$ of each) in succession. The loaded cartridge was washed with water $(20 \mathrm{ml})$ followed by hexane $(8 \mathrm{ml})$, and eicosanoids were eluted with methanol $(8 \mathrm{ml})$. The collected methanol fraction was dried under a stream of $\mathrm{N}_{2}$, and the residue was resuspended again in methanol $(0.2 \mathrm{ml})$ for storage at $-80^{\circ} \mathrm{C}$ until analysis by liquid chromatography-tandem mass spectrometry (LC-MS/MS).

LC-MS/MS analysis. Eicosanoid identification and quantification was performed with a Q-TRAP 3200 linear ion trap quadrupole LC-MS/MS equipped with a Turbo $\mathrm{V}$ ion source, operated in negative electrospray mode (Applied Biosystems). Samples, which had been reduced to a small volume in methanol $(10 \mu \mathrm{l})$, were injected into HPLC via an Agilent 1200 standard series autosampler equipped with a thermostat set at $4^{\circ} \mathrm{C}$ (Agilent Technologies). The HPLC component consisted of an Agilent 1100 series binary gradient pump equipped with an Agilent Eclipse plus $\mathrm{C}_{18}$ column $(50 \times 4.6 \mathrm{~mm}, 1.8 \mu \mathrm{m})$ or a Luna $\mathrm{C}_{18}(2)$ column $(150 \times 2 \mathrm{~mm}, 5 \mu \mathrm{m})$ (Phenomenex, Torrance, CA). The column was eluted at a flow rate of $0.5 \mathrm{ml} / \mathrm{min}$ with a mobile phase of methanol-water-acetic acid (65:35:0.03 vol/vol/vol) and a gradient increasing to methanol $100 \%$. Multiple reaction monitoring was used with a dwell time of 25 or $50 \mathrm{~ms}$ for each compound and the following source parameters: ion spray voltage, $-4500 \mathrm{~V}$; curtain gas, 40 units; ion source gas flow rate, 1.65 and 2.5 units; temperature, $600^{\circ} \mathrm{C}$. For eicosanoid quantification, standard curves (5-500 pg) were constructed using synthetic reference compounds and the internal standard.

Analysis of data. With in vitro recording, baseline contractile tension refers to the net active tension (i.e., total tension minus applied tension) developed by the preparation before any treatment. Constrictor responses were measured by the rise in tension (in absolute values) over baseline, while relaxant responses were given as reversal of tension (in percent values) in the precontracted vessel.

Data are expressed as the means $\pm \mathrm{SE}$. Comparisons were made using a Student's $t$-test or ANOVA, followed by the Bonferroni test.
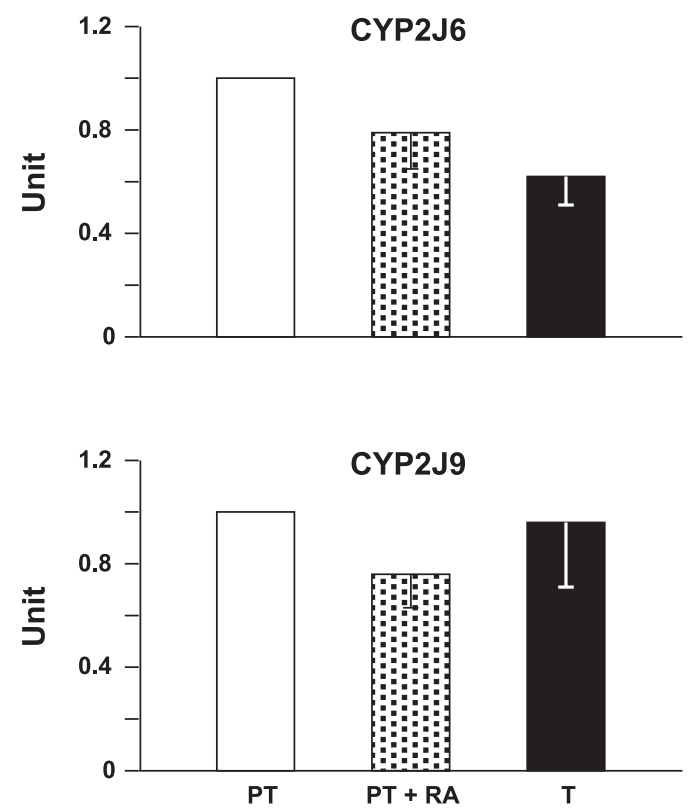

Fig. 1. Quantitative RT-PCR for CYP2J6 and CYP2J9 from the ductus arteriosus of the untreated preterm (PT) and term (T) mouse, and the preterm mouse treated with retinoic acid (PT+RA) (for details, see MATERIALS AND METHODS). With every condition, relative levels of transcript refer to five experiments (each in triplicate) and are expressed in arbitrary units after normalization by the reference $\beta$-actin. 
Kruskal Wallis test was also used, as required. Differences are considered significant for $P<0.05$. In those instances in which an eicosanoid was not measurable in the tissue incubate, a value equal to zero was taken for statistical computation.

\section{RESULTS}

Gene expression analysis. The ductus arteriosus presented a distinctive pattern of gene expression. As shown in Table 1, no mRNA was found for CYP450s belonging to the CYP2C subfamily, while CYP2J subfamily transcripts corresponding to CYP2J6 and CYP2J9 were present. Both transcripts were already evident in preterm mice and showed no change (CYP2J9), or a modest reduction (CYP2J6), with advancing gestation toward term or following treatment with retinoic acid (Fig. 1). Significantly, however, CYP2J6, unlike CYP2J9, is unable to metabolize AA (18) and cannot qualify as a source of EETs for EDHF function. Equally selective was the expression of genes for AA $\omega$-hydroxylation, with only CYP4A31 expressed in mouse ductus. CYP4B1 was also detected, but its function is largely in the synthesis of 12R-HETE rather than 20-HETE (19).

Localization of CYP2J6 and CYP2J9. Fluorescence microscopy showed CYP2J6 and CYP2J9 immunostaining in the ductus arteriosus of the term animal. Its distribution was diffuse with both proteins and encompassed intimal and medial layers (Fig. 2, $A$ and B). However, in the case of CYP2J6, a moderately higher intensity was noted in the intimal layer (Fig. $2 B$ ). Furthermore, through the use of appropriate markers, the staining proved to colocalize with the endoplasmic reticulum and not the plasma membrane. Figure 2, $D-F$, and Supplemental Fig. 2 illustrate the colocalization of, respectively, CYP2J9 and CYP2J6 with the endoplasmic reticulum in both muscle and endothelial cells. The ductus from the preterm mouse, whether untreated or retinoic acid treated, had a comparable pattern of immunoreactivity (Supplemental Fig. 3). In all cases, no immunostaining could be detected if the primary antibody was omitted (Fig. 2, Supplemental Fig. 3).

Eicosanoid release. EETs were formed in a variable amount by the ductus under basal conditions. As shown in Fig. 3, 5,6-EET was below detection in all but one of the samples, while the other EET regioisomers were measurable most of the times, and, among them, 14,15-EET and 11,12-EET were relatively more abundant. This pattern is consistent with the known regiochemical arrangement of CYP2J9, which makes mostly 14,15-EET and 11,12-EET (24). Furthermore, regardless of their basal value, EET levels did not increase upon treatment with compound 1555, the cocktail of inhibitors promoting EDHF formation, or the two procedures combined (Fig. 3). In fact, this profile remained essentially unchanged, even when using a fivefold larger pool of specimens for the analysis (Fig. 3). This negative result stands in contrast with the intrinsic potential of the AA epoxygenase for activation, as evidenced by the upward trend of EET formation when raising the oxygen concentration in the incubation medium from 2.5 to $30 \%(3.34 \pm 2.15,9.56 \pm 6.35$, and $11.12 \pm 4.31 \mathrm{pg} / \mu \mathrm{g}$ protein for, respectively, 8,9-, 11,12-, and 14,15-EET). Indeed,
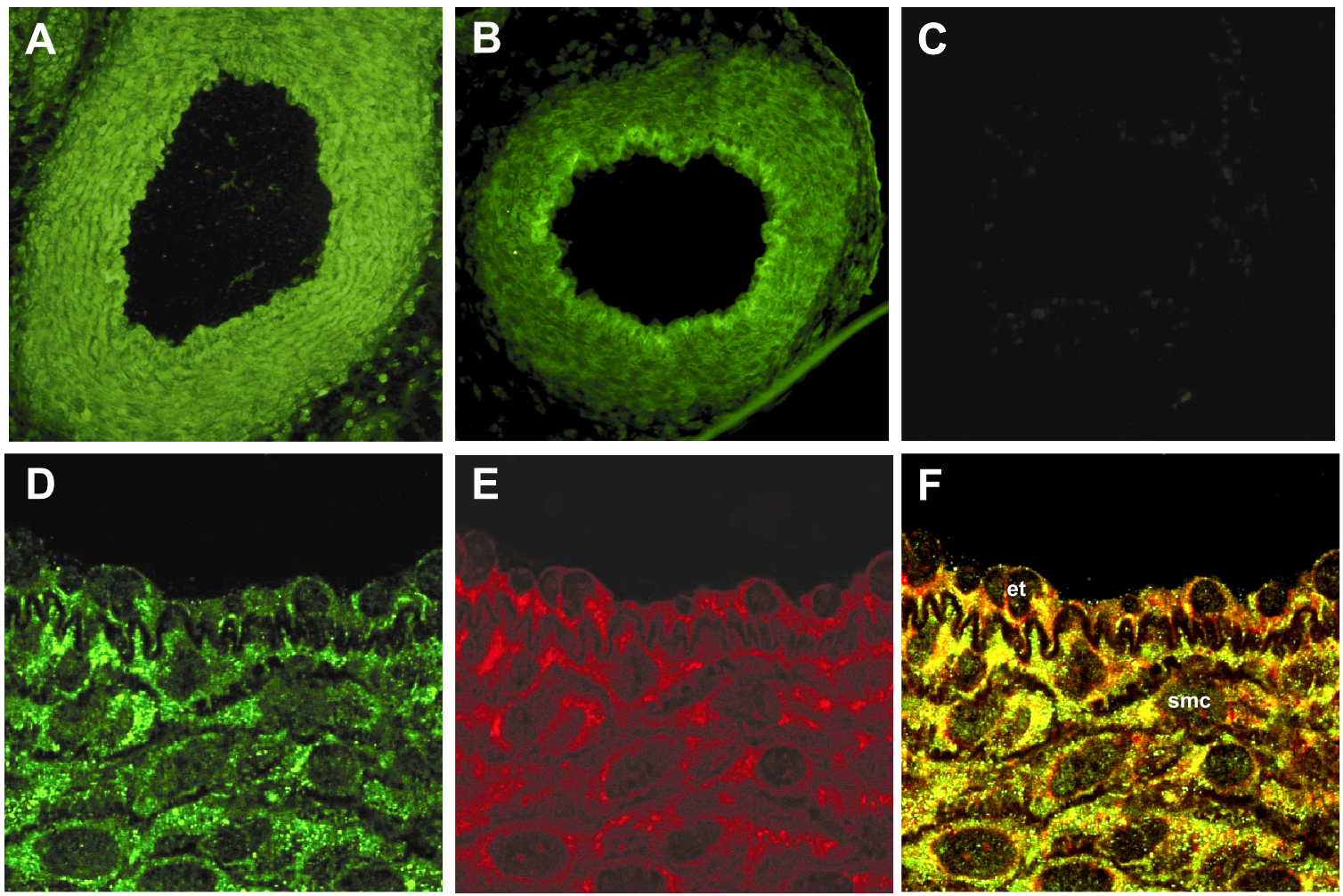

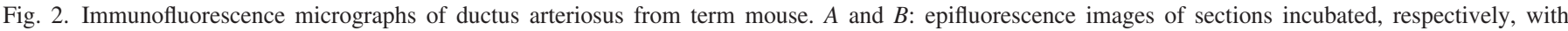

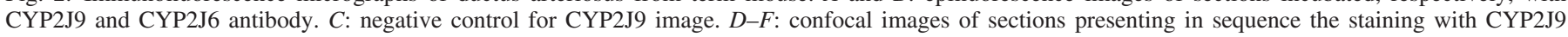

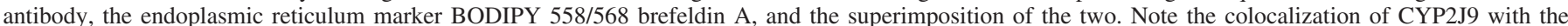

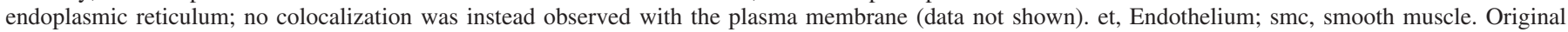
magnification: $\times 20(A-C)$ and $\times 40(D-F)$. 
significance of the oxygen response was barely missed in the case of 14,15-EET ( $t$ value of 2.11 when 2.16 is required for $P<0.05)$. Significantly in the present context, the yield of EETs showed no obvious reduction following removal of the endothelium (Fig. 3). On the contrary, there was a trend in the opposite direction (Fig. 3). As predicted from the finding with compound 1555 , none of the epoxide hydrolase products (i.e., 5,6-, 8,9-, 11,12-, and 14,15-di-HETrE) were detected, whether
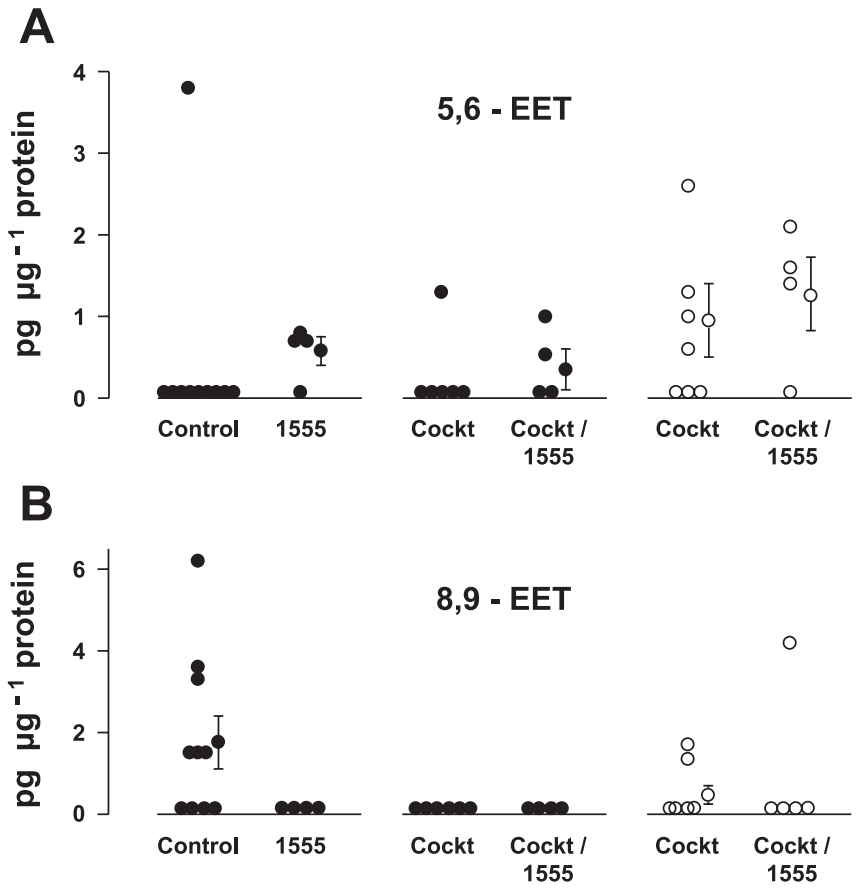

C
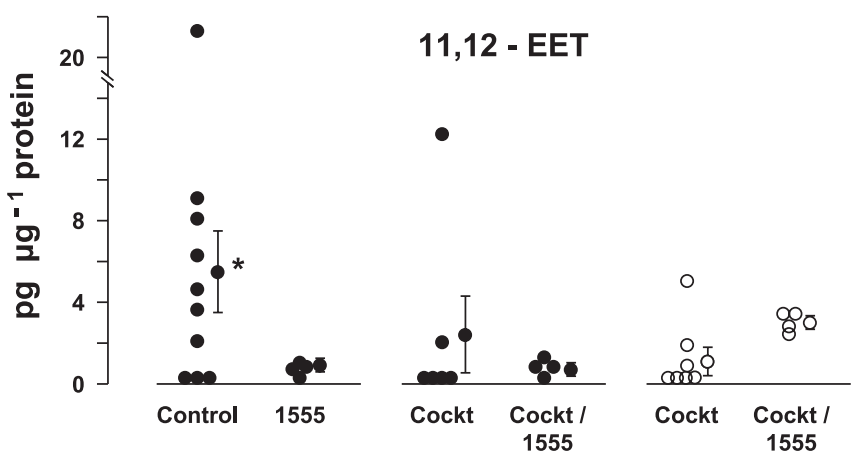

D

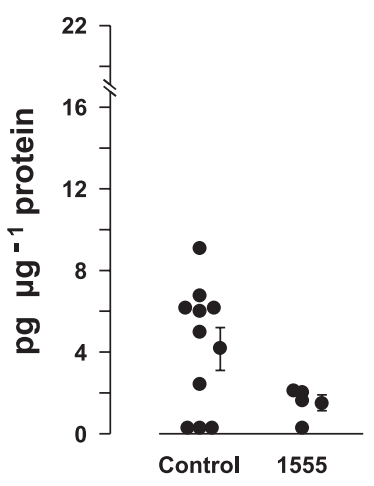

14,15 - EET

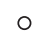

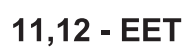
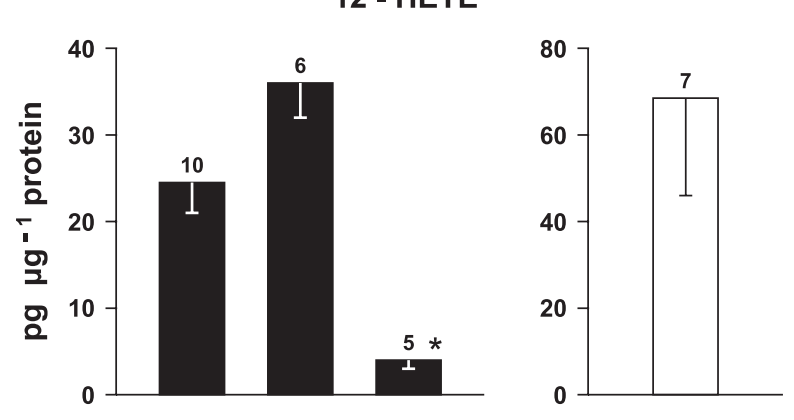

12 - HETrE
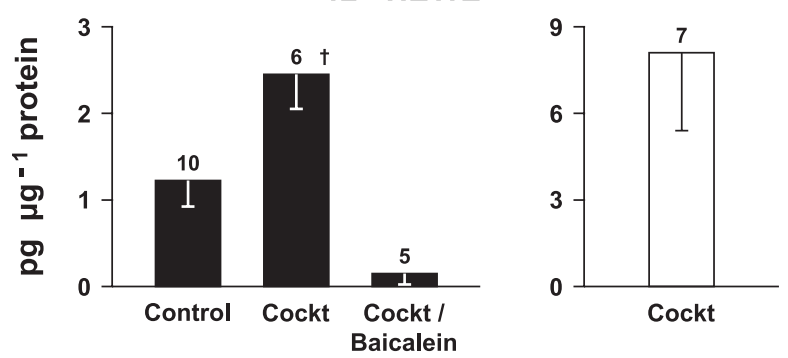

Fig. 4. Release of 12-hydroxytetraenoic acids (12-HETE) and its metabolite, 12-hydroxyeicosatrienoic (12-HETrE), from the intact (solid bars) and endothelium-denuded (open bars) term mouse ductus arteriosus under basal conditions and during treatment with a cocktail of inhibitors (Cockt), alone or with baicalein (for details, see MATERIALS AND METHODS). $* P<0.01$ and $P<0.001$ vs. control and cocktail groups, respectively; $\dagger P<0.05$ and $P<0.001$ vs. control and cocktail/baicalein groups, respectively. Note that differences between +endothelium/cocktail and -endothelium/cocktail for both compounds are not significant.

at rest or in the presence of the inhibitory cocktail, the only exception being a couple of samples from endothelium-denuded vessels treated with cocktail alone (14,15-di-HETrE, 1.7 $\mathrm{pg} / \mu \mathrm{g}$ protein) or cocktail plus compound 1555 (11,12-diHETrE, $0.2 \mathrm{pg} / \mu \mathrm{g}$ protein).

Contrary to EETs, 12-HETE was quite abundant in the ductus incubations, and its level rose upon treatment with the inhibitory cocktail (Fig. 4). Removal of the endothelium did not change this trend, although results were too variable for a firm conclusion (Fig. 4). An identical profile was noted with the 12-HETE metabolite, 12-HETrE, which was present at much lower levels (Fig. 4). Conversely, hepoxilin $\mathrm{A}_{3}$, a major additional product of $12 \mathrm{~S}$-lipoxygenase with distinct biological properties, was absent under all conditions, including experiments with a larger pool of specimens or with specimens exposed to $30 \%$ oxygen. Both 12-HETE and 12-HETrE levels

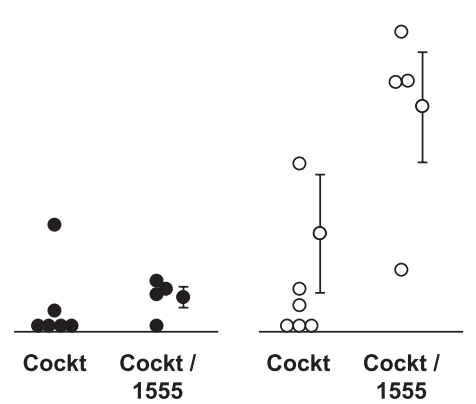

Fig. 3. Release of epoxyeicosatrienoic acids (EETs) from the intact (•) and endothelium-denuded $(\circ)$ term mouse ductus arteriosus under basal conditions and during treatment with either, or both, compound 1555 (epoxide hydrolase inhibitor) and a cocktail of inhibitors (Cockt; indomethacin, $N^{\mathrm{G}}$-nitro-Larginine methyl ester, zinc protoporphyrin) (for details, see MATERIALS AND METHODS). A: 5,6-EET. B: 8,9-EET. $C$ : 11,12-EET. $D$ : 14,15-EET. For each compound, differences among experimental conditions are not significant. Conversely, when comparing control values for different compounds, a significant difference $(* P<0.05)$ was found between 5,6-EET and 11,12-EET. Note that the analysis of a fivefold larger pool of specimens, treated with cocktail and compound 1555, yielded comparable results. With endothelium/ without endothelium (values in $\mathrm{pg} / \mu \mathrm{g}$ protein): 5,6-EET, 0.2/0.1; 8,9-EET, 0/0; 11,12-EET, 0.3/0.4; 14,15-EET, 0.4/0.5. 
were reduced to marginal values upon treatment with baicalein (Fig. 4). 5-HETE and 15-HETE were also present in small amounts in many of the samples, and their yield did not change significantly upon treatment with the inhibitory cocktail (Supplemental Fig. 4). Baicalein had no further effect on the cocktail-treated vessel, although a significant elevation over basal values was attained in the case of 5-HETE (Supplemental Fig. 4). Products of $\omega$-hydroxylation, specifically 18-, 19-, and 20-HETE, were not detected in all cases.

Study of bradykinin response. The isolated ductus arteriosus developed transient contractions $(0.1-0.8 \mathrm{mN} / \mathrm{mm})$ during the period of equilibration and then stabilized in most cases at the original baseline. Subsequent exposure to the inhibitory cocktail (indomethacin, L-NAME, ZnPP) produced an immediate and sustained contraction. Its magnitude was not affected to any significant degree by addition of baicalein $(10 \mu \mathrm{M})$, with final values of tension being $0.59 \pm 0.07$ and $0.93 \pm 0.14$ $\mathrm{mN} / \mathrm{mm}$, respectively, in the absence and presence of this drug. The reference $\mathrm{TxA}_{2}$ analog, ONO-11113 $(0.1 \mu \mathrm{M})$ gave an equivalent response $(0.83 \pm 0.11 \mathrm{mN} / \mathrm{mm}, n=5)$, indicating that the vessel had reached its maximal contractile potential. Bradykinin relaxed the precontracted ductus in a concentration-related manner, and no significant difference was noted in either the potency or pattern of the response due to baicalein (Fig. 5).

\section{DISCUSSION}

This study demonstrates that the ductus arteriosus is endowed with a CYP450 mechanism for the synthesis of EETs. Peculiarly, it appears to be limited to a single hemoprotein, CYP2J9, and lacks in particular a contribution from the CYP2C subfamily, which is conventionally regarded as a prime AA epoxygenase $(12,29)$ and, by extension, a potential EDHF synthase (11). Nonetheless, EETs are produced in the tissue, and their relative amount conforms to a general pattern (29), with 5,6-EET being less abundant compared with the other members of the group. This is consistent with the known regiochemical profile of EET formation by recombinant CYP2J9 (24). Their mode of formation, however, does not

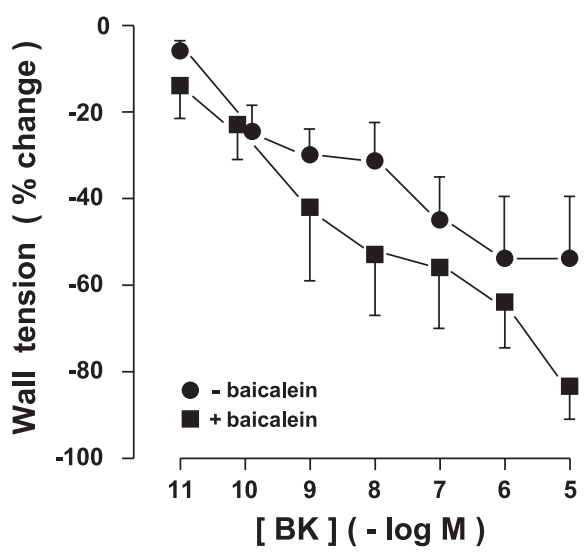

Fig. 5. Isolated ductus arteriosus from term fetal mouse. Concentrationresponse curves to bradykinin $(\mathrm{BK})$ in preparations precontracted with the combination indomethacin $(2.8 \mu \mathrm{M}) / N^{\mathrm{G}}$-nitro-L-arginine methyl ester (100 $\mu \mathrm{M})$ /zinc protoporphyrin $(10 \mu \mathrm{M})$, in the absence and presence of baicalein $(10 \mu \mathrm{M})(n=4$ for both groups) are shown. Wall tension before BK was as follows: without baicalein, $0.59 \pm 0.07 \mathrm{mN} / \mathrm{mm}$; with baicalein, $0.93 \pm 0.14$ $\mathrm{mN} / \mathrm{mm}$. Difference between the two curves is not significant. present the features that are expected for an EDHF agent. No obvious increase was noted when treating the ductus with a set of inhibitors that are known to promote EDHF formation (2). Furthermore, EET levels, however, modest and intrinsically variable, did not show any reduction upon removal of the endothelium. Endothelium dependence is a central attribute of the EDHF mechanism, in the ductus (2) as in other blood vessels (10). A methodological factor inherent to stability of CYP450 ex vivo, accessibility of the luminal surface of vessels to the incubation medium, or metabolism and reesterification of newly formed EETs, is unlikely to be responsible for this negative finding. CYP450 hemoprotein levels abate in vitro when lacking the solicitations of the in vivo environment, but this event unfolds over a time scale not applicable to our situation $(12,17)$. The caliber of the ductus is small indeed (see MATERIALS AND METHODS), but unimpeded equilibration between the intraluminal milieu and the bathing medium is still expected, thanks to the shortness of the vessel and the gentle shaking throughout the incubation. Metabolic systems, such as soluble epoxide hydrolase and COX, with the latter being particularly effective on 5,6-EET (12), cannot be considered for any interference, since tests were performed in the presence of appropriate inhibitors. Two possibilities remain as a potential confounding element: 1 ) EET removal through $\beta$-oxidation; and/or 2) EET incorporation into phospholipids $(12,29)$. Even such occurrence, however, seems remote, since EET formation was amenable to activation by oxygen and, by analogy, should have shown signs of an equivalent response under the influence of the inhibitory cocktail. It is reasonable to conclude, then, that a methodological factor cannot obscure the actual synthesis of EETs or any change expected from targeted manipulations. Hence, none of these compounds qualifies as an EDHF in the ductus. Our position, however, is not necessarily extendable to other vascular districts, specifically in the adult, where substantial evidence has been accrued for the EET mediation in $\operatorname{EDHF}(11,13)$. At the same time, the prospect remains open for alternative role(s) in the ductus of CYP2J9 and the AA-unrelated CYP2J6, as one gathers from their broad distribution across the vessel wall. In fact, CYP450 hemoproteins are a known constituent of blood vessels, within and without the endothelium, where they may contribute to a host of physiological and pathophysiological processes $(5,7,12,29)$.

While reaching this conclusion for the EETs, a question emerges on the postulated role of 12S-HETE as an EDHF (10, 36). This issue is intertwined with the possible involvement of the CYP450-derived 12R-HETE in the ductus. ${ }^{2}$ The latter compound may, in fact, yield the vasodilator 12R-HETrE at certain sites $(21,33)$ and cannot be distinguished from the $12 \mathrm{~S}$-enantiomer with our analytic procedures. In principle, either or both compounds could be formed in the ductus. The vessel has a highly expressed transcript for 12S-lipoxygenase (9), but, at the same time, is endowed with CYP4B1, which is linked with the synthesis of 12R-HETE (19). Furthermore, occurrence of 12-HETrE does not provide a clue on the identity of the parent compound, since it can be formed with equal ease from both enantiomers (33). Ductal 12S-lipoxygenase, on the other hand, seems peculiar in not generating hepoxilin $\mathrm{A}_{3}$, even

\footnotetext{
${ }^{2} 12 \mathrm{R}$-HETE may also be formed from $12 \mathrm{R}$-lipoxygenase, but this is a minor pathway being restricted to epithelial cells $(3,27)$.
} 
though hepoxilin synthase is an integral part of the enzyme (23). Hence, two interconnected questions may be raised. Does 12R-HETE contribute to the product being measured in the ductus incubate? Does 12S-HETE qualify as EDHF in the ductus? Based on our results, the answer is negative on both counts. Release of 12-HETE is blocked by baicalein, suggesting identification of the compound as the $12 \mathrm{~S}$-enantiomer. 12S-HETE, on the other hand, cannot act as EDHF, even though its greater yield upon treatment with the inhibitory cocktail would accord with such a role (see Fig. 4). Against this possibility is the persistence of the compound following removal of the endothelium and, even more significantly, the lack of any effect of baicalein on the relaxation of the ductus to bradykinin under conditions in which the response is entirely sustained by EDHF (2).

What is, then, the function of an active 12S-lipoxygenase pathway in the ductus? No information on this point is forthcoming from the current investigation. However, in the past, we have discussed the possibility of 12S-HETE promoting endothelial NO synthase and, hence, accounting for the enhanced NO-based relaxation secondary to COX inhibition (28). The present results are in accordance with this concept, inasmuch as they show an increase of 12-HETE synthesis during treatment with the inhibitory cocktail containing indomethacin. However, there may be a broader, hitherto undefined, impact of this pathway, and future research should consider this possibility.

In the end, the original question of the nature of EDHF in the ductus remains unanswered. Any further elaboration on this point is necessarily speculative, although the concept of EDHF originating via a CYP450 system that is normally inhibited by NO and CO remains firm (2). A possible clue on its identity is found in the recent characterization of two novel epoxygenasecatalyzed products of AA, 2-(11,12-epoxyeicosatrienoyl)glycerol and 2-(14,15-epoxyeicosatrienoyl)glycerol (6). Both compounds are potent vasodilators, and their synthesis is increased by bradykinin (6). Alternatively, if one moves to a different class of agents, it is intriguing to note an apparent coincidence between the prospective EDHF and the characteristics of hydrogen sulfide $\left(\mathrm{H}_{2} \mathrm{~S}\right)$ as they emerge from recent studies. $\mathrm{H}_{2} \mathrm{~S}$ is suitably effective as a vasodilator, and, moreover, it is formed in the endothelium via heme-containing enzymes being inhibited by both $\mathrm{NO}$ and $\mathrm{CO}(15,16,34)$. Further work will be required to verify either possibility.

A final point deserves a comment and relates to the absence in the ductus of products of AA $\omega$-hydroxylation, in particular 20-HETE. This finding is surprising in view of the occurrence of a relevant hemoprotein (CYP4A31) and the broad role as a relaxant or contractile agent being assigned to 20-HETE in the adult vasculature $(5,20)$. Conceivably, CYP450-based AA metabolic pathways develop in a differential manner, and the functional implications of such arrangement remain to be defined.

In conclusion, we have shown that the ductus arteriosus is endowed with the AA epoxygenase, but not the AA $\omega$-hydroxylation, pathway. AA epoxygenase develops at an early stage of gestation, and the lifespan of its EET products is not conditioned by epoxide hydrolase. Despite some probative data from the literature, none of the EETs appear to qualify as an EDHF in the ductus. 12S-lipoxygenase is a major AA metabolic pathway in the vessel, but, once again at variance with prior evidence, it does not contribute to the EDHF mechanism. The issue of the identity of EDHF in the ductus remains open, but this search is being narrowed to fewer possibilities.

\section{GRANTS}

This work was supported by grants of the Italian Ministry of Education and Research (PRIN 2007E7Y7R to F. Coceani) and the National Institutes of Health (NIH) (Grant HL34300 to M. L. Schwartzman). Portions of this work were also supported by the Intramural Research Program of the NIH, National Institute of Environmental Health Sciences (Z01 ES025034). S. Nencioni and D. Sodini are recipient of, respectively, a graduate studentship and a postdoctoral fellowship from the Scuola Superiore Sant'Anna.

\section{DISCLOSURES}

No conflicts of interest are declared by the author(s).

\section{REFERENCES}

1. Baragatti B, Brizzi F, Ackerley C, Barogi S, Ballou LR, Coceani F. Cyclooxygenase-1 and cyclooxygenase- 2 in the mouse ductus arteriosus: individual activity and functional coupling with nitric oxide synthase. $\mathrm{BrJ}$ Pharmacol 139: 1505-1515, 2003.

2. Baragatti B, Brizzi F, Barogi S, Laubach VE, Sodini D, Shesely EG, Regan RF, Coceani F. Interactions between NO, CO and an endotheliumderived hyperpolarizing factor (EDHF) in maintaining patency of the ductus arteriosus in the mouse. Br J Pharmacol 151: 54-62, 2007.

3. Boeglin WE, Kim RB, Brash AR. A 12R-lipoxygenase in human skin: mechanistic evidence, molecular cloning, and expression. Proc Natl Acad Sci USA 95: 6744-6749, 1998.

4. Campbell WB, Falck JR. Arachidonic acid metabolites as endotheliumderived hyperpolarizing factors. Hypertension 49: 590-596, 2007.

5. Capdevila JH, Falck JR, Harris RC. Cytochrome P450 and arachidonic acid bioactivation. Molecular and functional properties of the arachidonate monooxygenase. J Lipid Res 41: 163-181, 2000.

6. Chen JK, Chen J, Imig JD, Wei S, Hachey DL, Guthi JS, Falck JR, Capdevila JH, Harris RC. Identification of novel endogenous cytochrome p450 arachidonate metabolites with high affinity for cannabinoid receptors. J Biol Chem 283: 24514-24524, 2008.

7. Coceani F. Cytochrome P450 in the contractile tone of the ductus arteriosus: regulatory and effector mechanisms. In: The Fetal and Neonatal Pulmonary Circulations, edited by Weir EK, Archer SL, and Reeves JT. Armonk, NY: Futura, 2000, p. 331-341.

8. Coceani F, Liu YA, Seidlitz E, Kelsey L, Kuwaki T, Ackerley C, Yanagisawa M. Endothelin A receptor is necessary for $\mathrm{O}_{2}$ constriction but not closure of ductus arteriosus. Am J Physiol Heart Circ Physiol 277: H1521-H1531, 1999.

9. Costa M, Barogi S, Socci ND, Angeloni D, Maffei M, Baragatti B, Chiellini C, Grasso E, Coceani F. Gene expression in ductus arteriosus and aorta: comparison of birth and oxygen effects. Physiol Genomics 25: 250-262, 2006.

10. Félétou M, Vanhoutte PM. Endothelium-dependent hyperpolarizations: past beliefs and present facts. Ann Med 39: 495-516, 2007.

11. Fisslthaler B, Popp R, Kiss L, Potente M, Harder DR, Fleming I, Busse R. Cytochrome P450 2C is an EDHF synthase in coronary arteries. Nature 401: 493-497, 1999.

12. Fleming I. Vascular cytochrome p450 enzymes: physiology and pathophysiology. Trends Cardiovasc Med 18: 20-25, 2008.

13. Huang A, Sun D, Jacobson A, Carroll MA, Falck JR, Kaley G. Epoxyeicosatrienoic acids are released to mediate shear-stress-dependent hyperpolarization of arteriolar smooth muscle. Circ Res 96: 376-383, 2005.

14. Hwang SH, Tsai HJ, Liu JY, Morisseau C, Hammock BD. Orally bioavailable potent soluble epoxide hydrolase inhibitors. J Med Chem 50: 3825-3840, 2007.

15. Kamoun P. Endogenous production of hydrogen sulfide in mammals. Amino Acids 26: 243-254, 2004.

16. Lefer DJ. A new gaseous signaling molecule emerges: cardioprotective role of hydrogen sulfide. Proc Natl Acad Sci USA 104: 17907-17908, 2007.

17. Loot AE, Popp R, Fisslthaler B, Vriens J, Nilius B, Fleming I. Role of cytochrome P450-dependent transient receptor potential V4 activation in flow-induced vasodilatation. Cardiovasc Res 80: 445-452, 2008. 
18. Ma J, Bradbury JA, King L, Maronpot R, Davis LS, Breyer MD, Zeldin DC. Molecular cloning and characterization of mouse CYP2J6, an unstable cytochrome P450 isoform. Biochem Pharmacol 64: 1447-1460, 2002.

19. Mezentsev A, Mastyugin V, Seta F, Ashkar S, Kemp R, Reddy DS, Falck JR, Dunn MW, Laniado-Schwartzman M. Transfection of cytochrome P4504B1 into the cornea increases angiogenic activity of the limbal vessels. J Pharmacol Exp Ther 315: 42-50, 2005.

20. Morin C, Guibert C, Sirois M, Echave V, Gomes MM, Rousseau E. Effects of $\omega$-hydroxylase product on distal human pulmonary arteries. Am J Physiol Heart Circ Physiol 294: H1435-H1443, 2008.

21. Murphy RC, Falck JR, Lumin S, Yadagiri P, Zirrolli JA, Balazy M, Masferrer JL, Abraham NG, Schwartzman ML. 12(R)-hydroxyeicosatrienoic acid: a vasodilator cytochrome P-450-dependent arachidonate metabolite from the bovine corneal epithelium. J Biol Chem 263: $17197-$ 17202, 1988

22. Nguyen X, Wang MH, Reddy KM, Falck JR, Schwartzman ML. Kinetic profile of the rat CYP4A isoforms: arachidonic acid metabolism and isoform-specific inhibitors. Am J Physiol Regul Integr Comp Physiol 276: R1691-R1700, 1999.

23. Nigam S, Patabhiraman S, Ciccoli R, Ishdorj G, Schwarz K, Petrucev B, Kühn H, Haeggström JZ. The rat leukocyte-type 12-lipoxygenase exhibits an intrinsic hepoxilin $\mathrm{A}_{3}$ synthase activity. J Biol Chem 279: 29023-29030, 2004.

24. Qu W, Bradbury JA, Tsao CC, Maronpot R, Harry GJ, Parker CE, Davis LS, Breyer MD, Waalkes MP, Falck JR, Chen J, Rosenberg RL, Zeldin DC. Cytochrome P450 CYP2J9, a new mouse arachidonic acid $\omega-1$ hydroxylase predominantly expressed in brain. J Biol Chem 276: 25467-25479, 2001

25. Ray WJ, Bain G, Yao M, Gottlieb DI. CYP26, a novel mammalian cytochrome P450, is induced by retinoic acid and defines a new family. J Biol Chem 272: 18702-18708, 1997.

26. Richard C, Gao J, LaFleur B, Christman BW, Anderson J, Brown N, Reese J. Patency of the preterm fetal ductus arteriosus is regulated by endothelial nitric oxide synthase and is independent of vasa vasorum in the mouse. Am J Physiol Regul Integr Comp Physiol 287: R652-R660, 2004.
27. Schneider C, Keeney DS, Boeglin WE, Brash AR. Detection and cellular localization of 12R-lipoxygenase in human tonsils. Arch Biochem Biophys 386: 268-274, 2001.

28. Sodini D, Baragatti B, Barogi S, Laubach VE, Coceani F. Indomethacin promotes nitric oxide function in the ductus arteriosus in the mouse. Br J Pharmacol 153: 1631-1640, 2008.

29. Spector AA, Norris AW. Action of epoxyeicosatrienoic acids on cellular function. Am J Physiol Cell Physiol 292: C996-C1012, 2007.

30. Wang MH, Brand-Schieber E, Zand BA, Nguyen X, Falck JR, Balu N, Laniado Schwartzman M. Cytochrome P450-derived arachidonic acid metabolism in the rat kidney: characterization of selective inhibitors. J Pharmacol Exp Ther 284: 966-973, 1998.

31. Wang Y, Mercer-Connolly A, Lines L, Toyoda O, Coceani F. Endothelium-denuded pulmonary resistance arteries from the fetal lamb: preparation and response to vasoactive agents. J Pharmacol Toxicol Methods 32: 85-91, 1994

32. Wu GR, Jing S, Momma K, Nakanishi T. The effect of vitamin A on contraction of the ductus arteriosus in fetal rat. Pediatr Res 49: 747-754, 2001.

33. Yamamoto S, Nishimura M, Conners MS, Stoltz RA, Falck JR, Chauhan K, Laniado-Schwartzman M. Oxidation and keto reduction of 12-hydroxy-5,8,10,14-eicosatetraenoic acids in bovine corneal epithelial microsomes. Biochim Biophys Acta 1210: 217-225, 1994.

34. Yang G, Wu L, Jiang B, Yang W, Qi J, Cao K, Meng Q, Mustafa AK, Mu W, Zhang S, Snyder SH, Wang R. $\mathrm{H}_{2} \mathrm{~S}$ as a physiologic vasorelaxant: hypertension in mice with deletion of cystathionine $\gamma$-lyase. Science 322: 587-590, 2008

35. Yokoyama U, Sato Y, Akaike T, Ishida S, Sawada J, Nagao T, Quan H, Jin M, Iwamoto M, Yokota S, Ishikawa Y, Minamisawa S. Maternal vitamin A alters gene profiles and structural maturation of the rat ductus arteriosus. Physiol Genomics 31: 139-157, 2007.

36. Zink MH, Oltman CL, Lu T, Katakam PV, Kaduce TL, Lee HC, Dellsperger KC, Spector AA, Myers PR, Weintraub NL. 12-Lipoxygenase in porcine coronary microcirculation: implications for coronary vasoregulation. Am J Physiol Heart Circ Physiol 280: H693-H704, 2001. 